\title{
REMARKS ON THE COMMUTATIVITY OF RINGS
}

\author{
M. F. SMILEY ${ }^{1}$
}

Introduction. A celebrated theorem of N. Jacobson [7] asserts that if (1) $x^{n(x)}=x$ for every $x$ in a ring $R$, where $n(x)$ is an integer greater than one, then $R$ is commutative. In a recent paper [2], I. N. Herstein has shown that it is enough to require that (1) holds for those $x$ in $R$ which are commutators: $x=[y, z]=y z-z y$ of two elements of $R$. The purpose of this note is to show that if $R$ has no nonzero nilpotent ideals, we may restrict $x$ in (1) to iterated commutators of any fixed degree. We also obtain a weaker result for arbitrary rings.

An important tool in the proof of our results is a lemma which generalizes a result of Kaplansky [4] to the effect that the only elements of a primitive ring which commute with all commutators are in the center. This tool is also useful in extending and complementing some results of Divinsky [1] on commuting isomorphisms of simple rings. These extensions include some recent results of Posner [9] on derivations in prime rings. As a final remark, we indicate an exceedingly brief proof of Herstein's result [3] on Jordan derivations in prime rings.

Although the proof of our first result follows that of Herstein very closely, we present a self-contained account for the convenience of the reader.

A generalization of Herstein's theorem. Before we state our theorem, let us introduce a bit of notation. In a ring $R$, define $L_{0}(R)=R$, and, inductively, $L_{k+1}(R)=\left\{[x, y] ; x \in R, y \in L_{k}(R)\right\}$ for each nonnegative integer $k$. We shall also use the notation $x D(y)=[x, y]$ for $x, y \in R$.

THEOREM 1. Let $k$ be an integer $\geqq 1$ and let $R$ be a ring in which (1) holds for every $x \in L_{k}(R)$. Then $L_{k}(R)=0$. If also $k=1$ or if $R$ has no nonzero nilpotent ideals, then $R$ is commutative.

CASE $1 . R$ is a division ring. Suppose that $u=[a, b] \neq 0$ for some $a \in R$ and some $b \in L_{k-1}(R)$. We have $u^{n}=u$ and $(\lambda u)^{m}=\lambda u$ for each

Presented to the Society January 28, 1958 under the title $A$ remark concerning the commutativity of rings; received by the editors February 4, 1958 and, in revised form, September 22, 1958.

1 The author wishes to express his thanks to Professor S. K. Berberian for a stimulating conversation which led to the present note, and also to a referee whose suggestion of the hypothesis: "no nilpotent ideals" generalized our lemma and shortened the proof of Theorem 1. 
$\lambda$ in the center $Z$ of $R$ and $m, n>1$. With $s=(n-1)(m-1)+1>1$, we obtain $u^{s}=u,(\lambda u)^{s}=\lambda u$ and it follows that $Z$ has characteristic $p \neq 0$ and each $\lambda \in Z$ is algebraic over the field $P$ of $p$ elements. If $u \in Z$, then $b u$ is not in $Z$ and $b[a, b]=[b a, b]$ may replace $u$ in the ensuing argument. Let $m(x)$ be the minimum polynomial of $u$ over $Z$ and let $F$ be the field obtained by adjoining the coefficients of $m(x)$ to $P$. Then $F$ is finite and has $q=p^{v}$ elements. Hence $u^{q}=u$ is impossible for the equation $x^{q}-x=0$ can have no more than $q$ roots in the field $F(u)$. Thus $u^{q} \neq u$. But $m\left(u^{q}\right)=(m(u))^{q}=0$ and hence $u^{q}=r^{-1} u r$ for some $r \in R\left[6\right.$, p. 46]. Set $z=D^{k}(u)=r\left(u-u^{q}\right)^{k} \in L_{k}(R)$. Then $P(u, z)$ is a finite division ring and $u z=z u$ by the little Wedderburn theorem. But $u z=z u$ yields $z u^{q}=z u, u^{q}=u$, a contradiction.

CASE 2. $R$ is a prime ring. Here $R$ has no nonzeronilpotent elements. For if $x^{2}=0$ with $x \in R$, let $r_{1}, \cdots, r_{k} \in R$. Then $y=x r_{1} x r_{2} x$ $\cdots x r_{k} x=x D\left(r_{1} x\right) D\left(r_{2} x\right) \cdots D\left(r_{k} x\right) \in L_{k}(R)$, and $y^{2}=0$ yields $y=0$. Using the primeness of $R k$ times, we get $x=0$. As usual, it follows that all idempotents of $R$ are in the center of $R$.

CASE 3. $R$ is a primitive (and hence prime) ring but $R$ is not a division ring. Let $\rho$ be a maximal right ideal of $R$ which contains no nonzero two-sided ideal of $R$. Clearly $\rho$ contains no nonzero central idempotents and hence, by Case $2, L_{k}(\rho)=0$. For $a \in \rho, r \in R, b \in L_{k-1}(\rho)$, we have $a r \in \rho$ and $[a r, b]=a[r, b]=0$. By the primeness of $R$ and the fact that $\rho \neq 0$, we find that $b \in Z \cap \rho=0$ and it follows that $L_{k-1}(\rho)=0$. By induction we obtain $L_{0}(\rho)=\rho=0$. Hence $R$ is a division ring, contrary to hypothesis.

CASE 4. $R$ is an arbitrary ring. If $N$ is the Jacobson radical of $R$, then $R / N$ is a subdirect sum of primitive rings which satisfy the condition of the theorem. By Cases 3 and $1, L_{k}(R / N)=0$. If $t \in L_{k}(R)$, then $t+N \in L_{k}(R / N)=0, t \in N, t^{n-1}=0$ (being an idempotent of $N$ ), $t=t^{n}=0$, as desired.

The proof of Theorem 1 will be completed by means of the following

Lemma. Let $R$ be a ring with no nonzero nilpotent ideals. Let $a \in R$ and let $k$ be a non-negative integer. Then $[a, t]=0$ for every $t \in L_{k}(R)$ if and only if $a$ is in the center of $R$.

Proof. The lemma is clear if $k=0$. We use induction on $k$. Let $u \in L_{k-1}(R), x \in R$, and compute

$$
\begin{aligned}
{[a, u][a, x] } & =[a,[a, u] x]-[a,[a, u]] x \\
& =[a,[a x, u]-a[x, u]] \\
& =-a[a,[x, u]]=0 .
\end{aligned}
$$


Using this result, we obtain

$$
0=[a, u][a, x u]=[a, u] x[a, u]+[a, u][a, x] u=[a, u] x[a, u] .
$$

It follows that $[a, u]=0$ since $[a, u]$ generates a nilpotent ideal. By our inductive hypothesis, $a$ is in the center of $R$. The converse is trivial.

The ring $R$ of all $k$ by $k$ upper triangular nilpotent matrices over a field has $L_{k-1}(R)=0$, but $R$ is commutative only if $k \leqq 2$.

Some remarks on related results. We shall now turn to our generalization [10] of the work [1] of Divinsky.

THEOREM 2. Let $a \rightarrow a^{\prime}$ be a nontrivial endomorphism of a prime ring $R$ onto $R$ and let (2) $\left[a, a^{\prime}\right]=0$ for all $a \in R$. Then $R$ is commutative.

Proof. By linearity, $\left[a^{\prime}, b\right]=\left[a, b^{\prime}\right]$ for all $a, b \in R$. Hence $\left[a^{\prime} b^{\prime}, c\right]$ $=a^{\prime}\left[b^{\prime}, c\right]+\left[a^{\prime}, c\right] b^{\prime}=\left[a b, c^{\prime}\right]=a\left[b, c^{\prime}\right]+\left[a, c^{\prime}\right] b$ and we have $\left(a^{\prime}-a\right)\left[b, c^{\prime}\right]+\left[a, c^{\prime}\right]\left(b^{\prime}-b\right)=0$ for all $a, b, c \in R$. Set $c=b$ and find that $\left[a, b^{\prime}\right]\left(b^{\prime}-b\right)=0$ for all $a, b \in R$. Then $0=\left[a x, b^{\prime}\right]\left(b^{\prime}-b\right)$ $=\left[a, b^{\prime}\right] x\left(b^{\prime}-b\right)$ yields $\left[a, b^{\prime}\right]=0$ or $b^{\prime}=b$ for all $a, b \in R$. If $w \in R-Z$, then $\left[a, w^{\prime}\right]=\left[a^{\prime}, w\right] \neq 0$ for some $a \in R$ (because the mapping ' is onto $R$ ), and it follows that $w=w$ '. Additivity of the mapping' forces $a^{\prime}=a$ for all $a \in R$, contrary to assumption. Thus $R-Z$ is empty and $R$ is commutative.

THEOREM 3. Let $a \rightarrow a^{\prime}$ be an anti-endomorphism of a ring $R$ onto $R$ and let $\left[a, a^{\prime}\right]=0$ for all $a \in R$. Let $R$ have no nonzero nilpotent ideals. Then every $x \in R$ satisfies $x^{2}=c x+d$ with $c$ and $d$ in the center $Z$ of $R$.

Proof. As before, $\left[a, b^{\prime}\right]=\left[a^{\prime}, b\right]$ for all $a, b \in R$, and we obtain (3) $a\left[x, b^{\prime}\right]+\left[a, b^{\prime}\right] x=x^{\prime}\left[a^{\prime}, b\right]+\left[x^{\prime}, b\right] a^{\prime}$ for all $a, b, x \in R$. Set $x=b$ and get (4) $\left[a, b^{\prime}\right] b=b^{\prime}\left[a^{\prime}, b\right]$ for all $a, b \in R$. By linearity, $\left[a, b^{\prime}\right] x$ $+\left[a, x^{\prime}\right] b=b^{\prime}\left[a^{\prime}, x\right]+x^{\prime}\left[a^{\prime}, b\right]$ for all $a, b, x \in R$. Interchange $a$ and $b$ and add to (3) to find that $\left[a,\left[x, b^{\prime}\right]\right]=-\left[a^{\prime},\left[x, b^{\prime}\right]\right]$ or $\left[a+a^{\prime}\right.$, $\left.\left[x, b^{\prime}\right]\right]=0$ for all $a, b, x \in R$. By the Lemma, $a+a^{\prime} \in Z$. Now $\left[a^{\prime} a, x\right]$ $=a^{\prime}[a, x]+\left[a^{\prime}, x\right] a$, and use of (4) gives $\left[a^{\prime} a, x\right]=a^{\prime}\left[a, x+x^{\prime}\right]=0$ for all $a, x \in R$. Thus $a^{\prime} a \in Z$ and we have $a^{2}=\left(a+a^{\prime}\right) a-a^{\prime} a$ for all $a \in R$.

REMARK 1 . If we assume that the ring $R$ of Theorem 3 is primitive, a result of Kaplansky [5] assures us that $R$ is commutative or is a quaternion algebra over $Z$.

REMARK 2. In Theorems 2 and 3, we may assume only that (5) $\left[a, a^{\prime}\right] \in Z$ for every $a \in R$ and still reach the same conclusions. In Theorem 2, for example, linearity and (5) give $\left[\left[a a^{\prime}, a^{\prime}\right]+\left[a, a^{\prime} a^{\prime \prime}\right]\right.$, 
$\left.a^{\prime}\right]=0$. Expansion and use of $\left[\left[a, a^{\prime \prime}\right], a^{\prime}\right]=0$, valid by (5) and Jacobi's identity, give $\left[a, a^{\prime}\right]\left[a^{\prime \prime}, a^{\prime}\right]=0$, and hence $\left[a, a^{\prime}\right]=0$ since the mapping ' is onto. An analogous computation is used in the case of Theorem 3. (When $2 \neq 0$ in $R$ and $R$ is prime, we may dispose of both cases at once since (6) $\left[a^{2}, a^{\prime 2}\right]=2\left[a, a^{\prime}\right]\left(a a^{\prime}+a^{\prime} a\right) \in Z$ yields $\left[a, a^{\prime}\right]$ $=0$ or $a a^{\prime} \in Z,\left[a a^{\prime}, a\right]=a\left[a^{\prime}, a\right]=0,\left[a^{\prime}, a\right]=0$.)

Remark 3. Posner [9] has discussed a derivation $a \rightarrow a^{\prime}$ of a prime ring $R$ into $R$ which satisfies (2), or, more generally, (5). Such a derivation gives rise to a homomorphism

$$
a \rightarrow a^{*}=\left[\begin{array}{ll}
a & a^{\prime} \\
0 & a
\end{array}\right]
$$

of $R$ into a matric ring $R_{1}$ [6, p. 102]. We may regard $R$ as a subring of $R_{1}$ in an obvious way. Then (2) yields $\left[a, a^{*}\right]=0$ for all $a \in R$, and the proof of Theorem 2 gives $\left[a, b^{*}\right] x\left(b^{*}-b\right)=0$, which becomes $[a, b] x b^{\prime}=0$ for all $a, b, x \in R$. Thus (2) yields the commutativity of $R$ or the triviality of the derivation. When only (5) holds, we obtain $\left[a, a^{*}\right]$ in the center of $R_{1}$ for all $a \in R$. If $2 \neq 0$ in $R$, (6) shows that $a^{2}\left[a, a^{\prime}\right] \in Z,\left[a^{2}\left[a, a^{\prime}\right], a^{\prime}\right]=2\left[a, a^{\prime}\right]^{2}=0$, and (2) holds. When $2=0$ in $R$, a direct proof of (2) from (5) is as follows. By (5) and linearity, $\left[\left[a a^{\prime}, a^{\prime}\right]+\left[a,\left(a a^{\prime}\right)^{\prime}\right], a\right]=0$, or $-\left[a, a^{\prime}\right]^{2}+\left[a\left[a, a^{\prime \prime}\right], a\right]=0$. But (5) and linearity show that $\left[a^{\prime}, a^{\prime}\right]+\left[a, a^{\prime \prime}\right] \in Z$, and hence $\left[a, a^{\prime}\right]^{2}=0$ and (2) holds.

REMARK 4. Herstein [3] has discussed a Jordan derivation $a \rightarrow a^{\prime}$ of a prime ring $R$, i.e., an additive mapping of $R$ into $R$ for which the induced mapping $a \rightarrow a^{*}$ of Remark 3 satisfies $\left(a^{2}\right)^{*}=\left(a^{*}\right)^{2},(a b a)^{*}$ $=a^{*} b^{*} a^{*}$ for all $a, b \in R$ and is, therefore, a Jordan homomorphism of $R$ into $R_{1}$. With $a^{b}=(a b)^{*}-a^{*} b^{*}$ and $a_{b}=(a b)^{*}-b^{*} a^{*}$, we have

$$
a_{b} a^{b}=a^{b} a_{b}=a_{b} r^{*} a^{b}+a^{b} r^{*} a_{b}=0
$$

for all $a, b, r \in R$. (See $[11 ; 12]$.) With $\{a, b\}=(a b)^{\prime}-\left(a b^{\prime}+a^{\prime} b\right)$, the identities (7) become

$$
[a, b]\{a, b\}=\{a, b\}[a, b]=[a, b] r\{a, b\}+\{a, b\} r[a, b]=0
$$

for all $a, b, r \in R$. The simple linearization argument of $\$ 2$ of [11] yields that $R$ is commutative or ' is an ordinary derivation of $R$. It is an easy consequence that a ring in which $2 x=0$ implies $x=0$ and in which zero is the only nilpotent ideal has no Jordan derivation which is not an ordinary derivation. However, it is possible that the Lie ring of Jordan derivations of a ring may yield some information about radical rings. 


\section{REFERENCES}

1. N. Divinsky, On commuting automorphisms of rings, Trans. Royal Soc. Canada, Sect. III vol. 49 (1955) pp. 19-22.

2. I. N. Herstein, $A$ condition for the commutativity of rings, Canad. J. Math. vol. 9 (1957) pp. 583-586.

3. - Jordan derivations of prime rings, Proc. Amer. Math. Soc. vol. 8 (1957) pp. 1104-1110.

4. I. Kaplansky, Semi-automorphisms of rings, Duke Math. J. vol. 14 (1947) pp. $521-525$.

5. —_ Rings with a polynomial identity, Bull. Amer. Math. Soc. vol. 54 (1948) pp. $575-580$.

6. N. Jacobson, The theory of rings, New York, 1943.

7. - Structure theory for algebraic algebras of bounded degree, Ann. of Math. vol. 46 (1945) pp. 695-707.

8. N. H. McCoy, Prime ideals in general rings, Amer. J. Math. vol. 71 (1949) pp. 823-833.

9. E. C. Posner, Derivations in prime rings, Proc. Amer. Math. Soc. vol. 8 (1957) pp. 1093-1100.

10. M. F. Smiley, Remarks on commuting automorphisms, (Abstract) Amer. Math. Monthly vol. 63 (1956) p. 535.

11. - Jordan homomorphisms onto prime rings, Trans. Amer. Math. Soc. vol. 84 (1957) pp. 426-429.

12. K. Yamaguti, Jordan and Jordan triple isomorphisms of rings, J. Sci. Hiroshima Univ. Ser. A vol. 20 (1957) pp. 107-110.

The State University of Iowa 